\title{
Immunohistochemical localization of dentin matrix protein 1 in human dentin
}

\author{
G. Orsini, ${ }^{1}$ A. Ruggeri, ${ }^{2}$ A. Mazzoni, ${ }^{2}$ F. Nato, ${ }^{2}$ M. Falconi, ${ }^{2}$ A. Putignano, ${ }^{1}$ \\ R. Di Lenarda, ${ }^{3}$ A. Nanci, ${ }^{4}$ L. Breschi ${ }^{3,5}$
}

${ }^{1}$ Department of Restorative Dentistry, Institute of Stomatology, Polytechnique University of Marche, Ancona, Italy; ${ }^{2}$ Department of SAU\&FAL, University of Bologna, Italy; ${ }^{3}$ Unit of Dental Sciences and Biomaterials, Department of Biomedicine, University of Trieste, Italy; ${ }^{4}$ Faculty of Dentistry, University of Montréal, Canada; ${ }^{5}$ GM-CNR, Unit of Bologna c/o IOR, Bologna, Italy

(C)2008 European Journal of Histochemistry

Dentin matrix protein 1 (DMP1) is a non-collagenous matrix protein with a recognized role in the formation of mineralized tissues such as dentin. The aim of this study was to analyze the distribution of DMP1 in human dentin by means of immunofluorescence and high-resolution immunogold labeling. Fully developed, sound human dentin specimens were submitted to fluorescence labeling and post-embedding immunolabeling techniques with a rabbit polyclonal antihuman DMP1 antibody followed by corresponding fluorochrome-conjugated or gold-conjugated secondary antibodies. Both immunofluorescence and immunogold labeling showed an intense labeling associated with the peritubular dentin. In addition, at the ultrastructural level, there was also a moderate and diffuse immunoreaction over intertubular dentin, and a weak labeling within predentin which increased in density towards the mineralization front. This study suggests that in adult human teeth, like in rodents, DMP1 is prevalently concentrated at the level of peritubular dentin and this feature is preserved also in fully developed-teeth. These data are consistent with what has been observed in rodents and suggest that DMP1 plays a role in maintenance of the dentin tubular space.

Key words: dentin, DMP1, immunohistochemistry,

fluorescence microscopy, transmission electron microscopy.

Correspondence: Lorenzo Breschi,

Division of Dental Sciences and Biomaterials,

Department of Biomedicine, University of Trieste,

Via Stuparich, 1, 34129 Trieste, Italy

Tel.: +39.040.3992192.

Fax: +39.040.662744.

E-mail: Ibreschi@units.it

Paper accepted on September 19, 2008

European Journal of Histochemistry

2008; vol. 52 issue 4 (October-December): 215-220
D entin matrix protein 1 (DMPI) is a multifunctional protein, prominent member of one category of non-collagenous proteins (NCPs), termed the SIBLINGS (Small IntegrinBinding Ligand, N-linked Glycoprotein), that regulates cell attachment and differentiation (Kulkarni et al. 2000; Narayanan et al. 2003), and plays a pivotal role in biomineralization (Ye et al. 2004, reviewed in Qin et al. 2007). George et al. (1993) identified DMPI by CDNA cloning and showed that it induces differentiation of mesenchymal cells into odontoblasts, and promotes mineralization. More recently, DMPI was found to induce cytodifferentiation of dental pulp stem cells into odontoblast-like cells during normal and pathological dentinogenesis, indicating that it could act as a morphogen with the potential to regenerate dentin-like tissue and to form reparative dentin (Almushayt et al. 2006). DMPl has been proposed to possess dual functions, both as a transcription factor that targeted the nucleus and as an extracellular matrix protein that initiated mineralization (Narayanan et al. 2003; 2006). Cooperative interactions between DMPI and type I collagen can initiate apatite nucleation and mineral deposition (He \& George 2004, He et al. 2005). Recently, in vivo studies by Lu and coworkers ( Lu et al. 2007) revealed that DMP1-null mice show abnormalities in mineralization and structural organization of dentin.

Studies in rodents have shown that DMPI is present in the nucleus and Golgi apparatus of odontoblasts, at the mineralization front, and in mineralized dentin (Massa et al. 2005). It immunolocalizes preferentially along the wall of dentinal tubules and in peritubular dentin (Massa et al. 2005; Lu et al. 2007). Recently, two studies on hypophosphatemic teeth of children have ana- 
lyzed presence of DMPI in dentin by means of immunohistochemistry, showing that DMPl, like other NCPs, was localized in the inner third of circumpulpal dentin, associated with the tubular and intertubular dentin, whereas in hypophosphatemic teeth DMPI was only observed in the dentin interglobular spaces (Boukpessi et al. 2006; Chaussain-Miller et al. 2007). To our knowledge the ultrastructural distribution of DMPI in human adult teeth has not yet been well elucidated. Since knowledge of the structure-function relationships is fundamental for the development of predictable regenerative therapies, we have applied immunofluorescence and post-embedding colloidal-gold immunolabeling to identify DMPI and determine its localization in human sound dentin.

\section{Materials and Methods}

Ten human third molars extracted as part of the orthodontic treatment plan were used in this study. The subjects had a mean age of $27.6 \pm 4.8$ years. The consent form and experimental protocol was approved by the Ethics Committee of the University of Bologna (Italy). Following extraction, roots were cut off with a low speed diamond saw and each tooth crown was then transversally sectioned to obtain $2 \mathrm{~mm}$-thick dentin disks at a medial level of the molar pulp chamber. The disks were immediately immersed in $4 \%$ paraformaldehyde and $0.1 \%$ glutaraldehyde buffered with 0.1 $\mathrm{M}$ sodium cacodylate, at $\mathrm{pH} 7.2$, and left overnight in the fixative at $4^{\circ} \mathrm{C}$.

\section{Fluorescence labeling}

For immunofluorescence labeling, the fixed disks were rinsed three times (10 min. each) in $0.1 \mathrm{M}$ sodium cacodylate, at $\mathrm{pH} 7.2$, Then, 100 $\mu \mathrm{m}$ sections were prepared using a high-precision cutting machine equipped with diamond disks (Remet, Bologna, Italy). Sections were immediately processed for incubation with a rabbit polyclonal anti-human DMPI antibody (LF-160, generously donated by Dr. Larry Fisher, National Institute of Dental and Craniofacial Research, Bethesda, MD, USA; Jain A et al. 2002; Fisher and Fedarko 2003) as follows. Sections were blocked with normal goat serum for 30 min., and incubated with a 1:100 dilution of the antibody

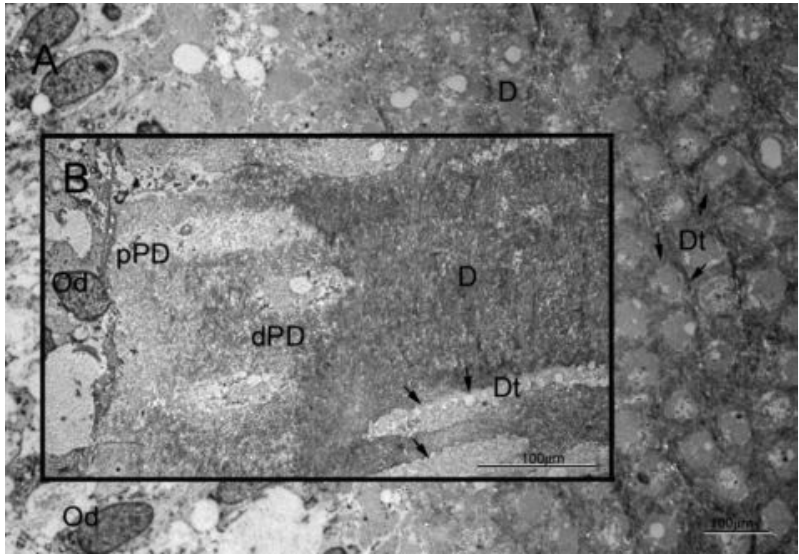

Figure 1. Transmission electron micrographs at low magnification of the examined human dentin. (A) illustrates the morphology of the dentin region (D) within the first 1000-1500 $\mu \mathrm{m}$ from the odontoblasts $(\mathrm{Od})$, in which cross-sections of dentinal tubules (Dt) are present (arrows). (B) shows the predentin region which can be distinguished in proximal predentin ( $p P D)$, near odontoblasts, and distal predentin (dPD), towards the mineralization front. In the initial layer of the dentin (D), longitudinal-cut dentinal tubules (Dt) were visible (arrows). Magnification bars: $100 \mu \mathrm{m}$.

(prepared in $0.05 \mathrm{M}$ Tris $\mathrm{HCl}$ buffer, TBS, at $\mathrm{pH}$ 7.6), overnight at $4^{\circ} \mathrm{C}$. After several TBS rinses, sections were subsequently incubated for 90 min., at room temperature and protected from light, with a goat anti-rabbit IgG diluted 1:100 coupled to Alexa Fluor 488 (Invitrogen S.r.l., Milan, Italy). The specimens were then washed for 10 min. in 0.05M TBS, briefly rinsed with distilled water, and coverslipped with aqueous mounting medium (Vecta-Shield, Vector Laboratories, Burlingame, CA, USA). Controls consisted of sections incubated (a) with secondary antibody only, (b) nonimmune serum. The sections were observed under an LSM 510 META confocal laser scanning microscope (Zeiss, Jena, Germany) at an excitation wavelength of $488 \mathrm{~nm}$.

\section{Immunocytochemical processing}

After fixation, disks were washed for $\mathrm{lh}$ in 0.1 $\mathrm{M}$ cacodylate buffer, $\mathrm{pH} 7.4$, and decalcified using $4.13 \%$ EDTA for three months at $4^{\circ} \mathrm{C}$. They were then extensively washed with $0.1 \mathrm{M}$ sodium cacodylate buffer, dehydrated in graded concentrations of ethanol and embedded in LR White resin (London Resin, Berkshire, UK). Semi-thin sections ( $1 \mu \mathrm{m})$ were cut with glass knives on a Reichert Jung Ultracut $\mathrm{E}$ ultramicrotome and 
stained with toluidine blue. Selected areas of the sample were trimmed for ultra thin sectioning ( 80 $n m$ ) using a diamond knife. Sections were mounted on Formvar carbon-coated nickel grids.

Grid-mounted tissue sections were processed for immunocytochemical labeling with anti-DMP1, as previously reported (Breschi et al. 2003; Suppa et al. 2006). In brief, sections immersed in 0.05 $\mathrm{M}$ Tris $\mathrm{HCl}$ buffered solutions (TBS) at $\mathrm{pH}$ 7.6, pre-incubated with normal goat serum for 30 min., and incubated overnight with anti-DMPl at $4^{\circ} \mathrm{C}$. Gold labeling was performed using goat IgG antirabbit IgG conjugated to $15 \mathrm{~nm}$ gold particles (British BioCell International, Cardiff, UK) diluted $1: 100$ in $0.02 \mathrm{M}$ Tris $\mathrm{HCl}$ buffered solutions (TBS) at $\mathrm{pH}$ 8.2. After labeling, grids were stained with $4 \%$ uranyl acetate and lead citrate for examination in a JEOL 1010 TEM operated at 60 kV (JEOL Ltd., Tokyo, Japan). The TEM was outfitted with a Digital Camera MegaView III operating under the Analysis Imaging System GmbH (Munster, Germany).

Controls consisted of sections incubated (a) with secondary antibody only; (b) substituting the primary antisera with nonimmune serum.

\section{Results}

Low magnification micrographs show that the sections analyzed were comprised between the odontoblasts layer lining the dental pulp and the first 1000-1500 $\mu \mathrm{m}$ of the dentin region (Figure $1 \mathrm{~A}, \mathrm{~B})$. In particular, Figure $1 \mathrm{~A}$ shows the localization of the dentinal tubules in a cross sectional view evaluated under immunofluorescence, in which DMPI localizes mainly to the peritubular dentin (Figure 2 A). Specimens incubated with nonimmune serum or secondary antibody only exhibited some background fluorescence but no significant labeling over peritubular dentin (Figure 2 B). Figure 1 B presents the thin predentin layer that is delimited by the odontoblast layer and the initial dentin, where a few longitudinal-cut dentinal tubules can be seen. At high magnification ultrastructural level, DMP1 immunolocalization showed an intense and circumscribed reactivity over the walls of the dentin tubules and a moderate and more diffuse labeling over intertubular dentin (Figure 3 A, B). A weak labeling was detected in predentin which increased
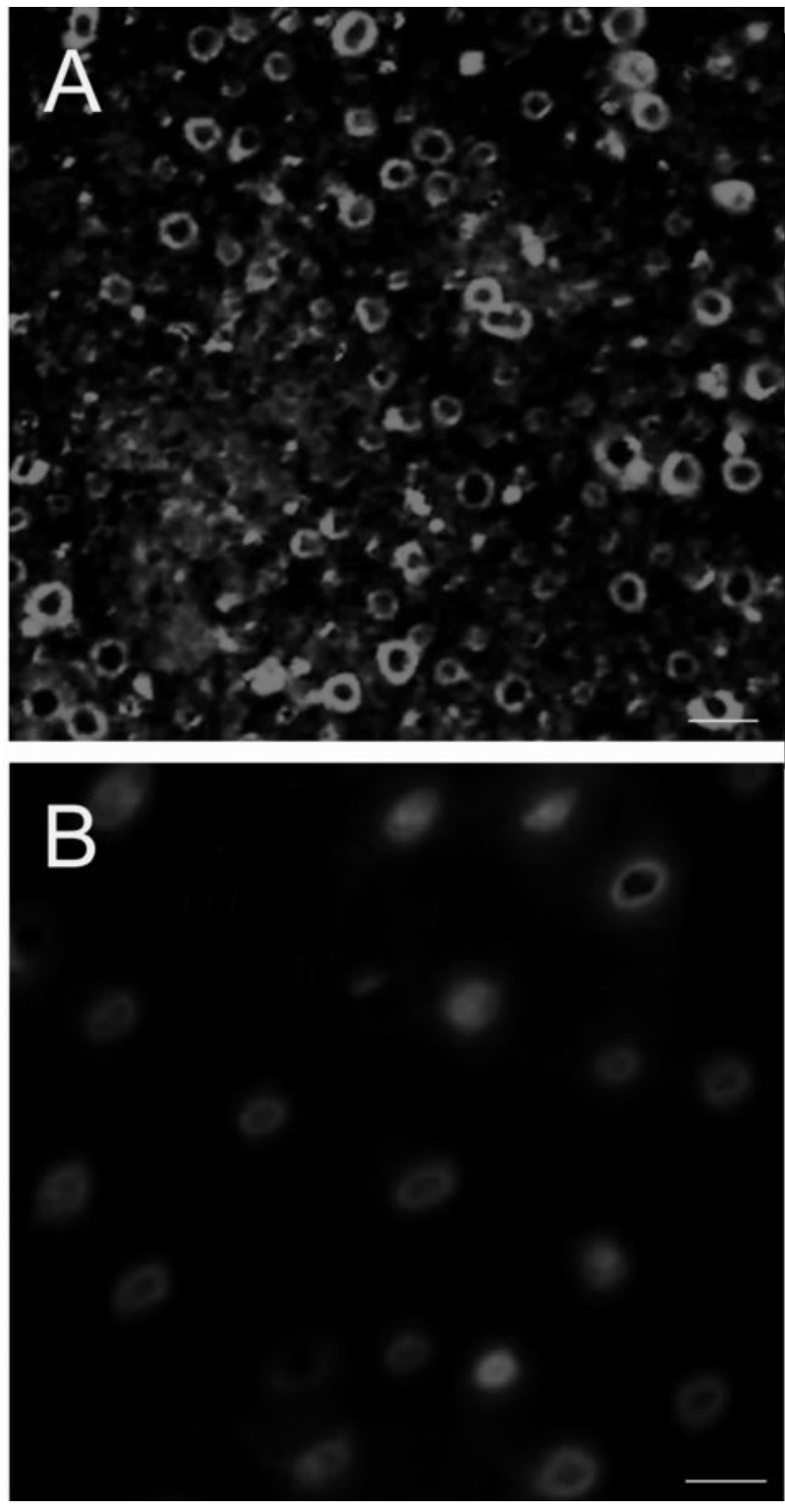

Figure 2. Image obtained with fluorescence microscope on human dentin. (A) Immunofluorescence labeling for DMP1 clearly showed the intense labeling over the peritubular dentin. (B) Control dentin incubated with nonimmune serum or secondary antibody only exhibited a few labeled dentin tubule walls. Magnification bars: 5 $\mu \mathrm{m}$

towards the mineralization front and which was more intense in regions where collagen fibrils are closely packed (Figure 3 C, D). A few gold particles were seen dispersed over the odontoblast bodies and processes (data not shown), but poor cellular preservation precluded assigning them unequivocally to specific subcellular compartments. Nuclei only revealed few scattered gold 

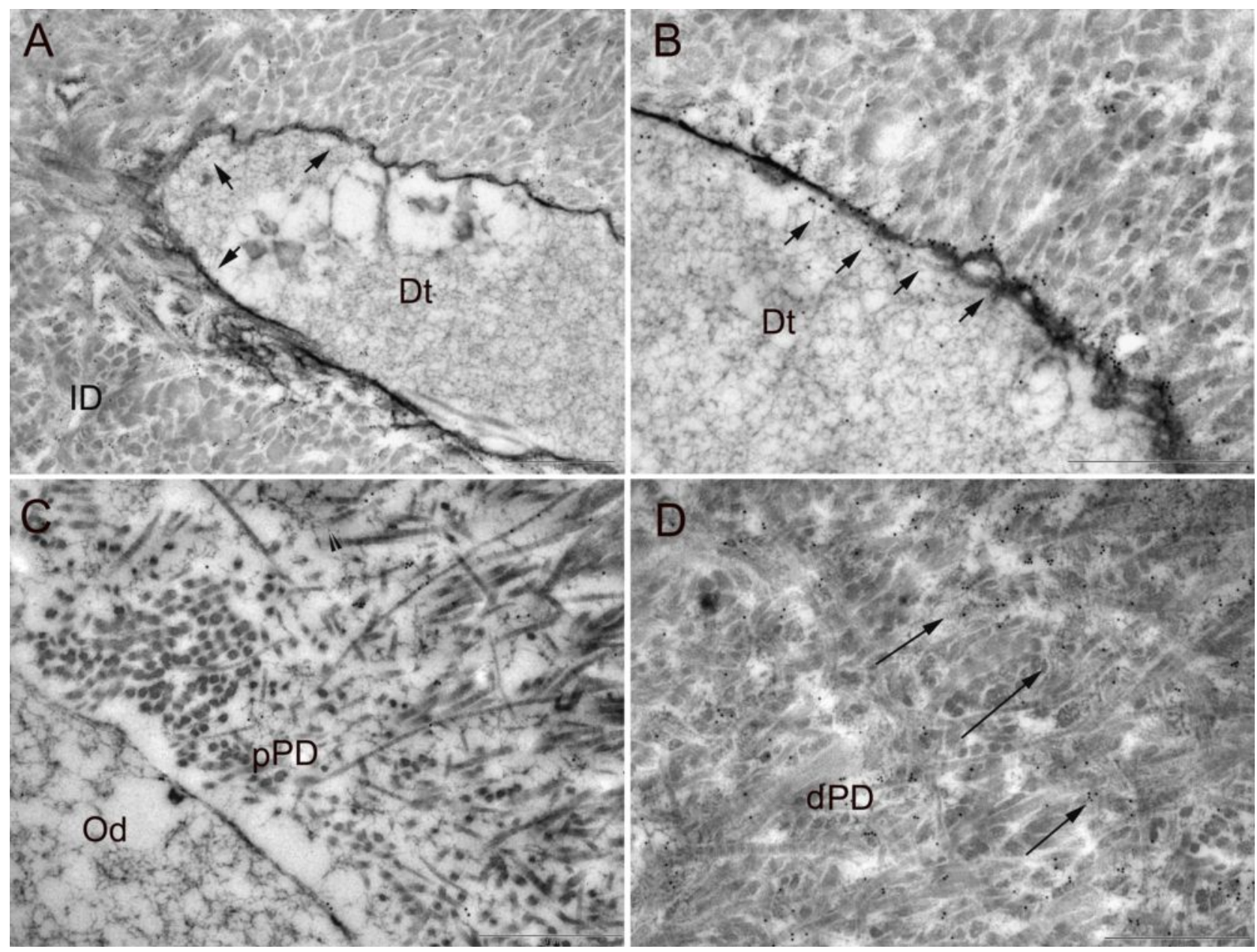

Figure 3. Transmission electron micrographs revealing different immunolabeling pattern of DMP1 on human dentin. (A) an intense and circumscribed immunoreactivity for DMP1 can be seen over the peritubular dentin (arrows) and a moderate and more diffuse labeling is evident over the intertubular dentin (ID); Dt: dentin tubule. (B) High magnification image of the intensely labeled walls of dentin tubules (arrows); Dt: dentin tubule. (C) A weak labeling for DMP1 is detectable in proximal predentin (pPD), near odontoblasts (Od). (D) The immunoreactivity for DMP1 increased in distal predentin (dPD), towards the mineralization front where collagen fibrils are closely packed (arrows). Magnification bars: $10 \mu \mathrm{m}$.

particles. Controls showed only few and randomly distributed gold particles over the tissue sections, thus confirming the specificity of the labeling (data not shown).

\section{Discussion}

Since the mechanism by which peritubular dentin forms and its precise composition are still not completely elucidated, it is most relevant to explore ultrastructural and immunohistochemical features of peritubular dentin (Gotliv et al. 2006). While the immunohistochemical pattern of DMP1 has been clearly elucidated in mice dentin (Feng et al. 2003; Lu et al. 2007), no correlative TEM and fluorescence data have been available on human sound dentin. DMP1 accumulates along the peritubular dentin wall of human sound dentin, similarly to what has been previously reported in mice (Lu et al. 2007), thus suggesting a functional role in their formation and maintenance (Baba et al. 2004).

Peritubular dentin has been shown to be hypermineralized (about 40\% more than intertubular dentin) (Nanci 2007). Gotliv and co-workers (2006) also pointed out that bovine peritubular dentin differs form intertubular dentin not only on the degree of mineralization, but also in the amount and nature of mineral elements and aminoacids. Human peritubular dentin has been reported to contain phosphoproteins and acidic 
glycosylated proteins (Weiner et al. 1999, Chaussain-Miller et al. 2007).

Like other noncollagenous matrix proteins, such as osteopontin, osteocalcin and dentin sialophosphoprotein, DMPI has been shown to be involved in the remodelling process during osteogenesis and during pathological processes and/or external insults (Toyosawa et al. 2004). For instance, a specific role of DMPI during bone remodelling has been postulated by Toyosawa and co-workers during fracture healing (2004). Once the tooth formation is completed, dentinogenesis continues at a slower rate, resulting in secondary dentin production under physiological conditions, whereas tertiary dentin is formed in response to noxious external stimuli, being classified into reparative dentin and reactionary dentin (reviewed in Smith and Lesot 2001; Goldberg and Smith 2004; Moses et al. 2006). During the physiologic response there is a continuous deposition of peritubular dentin, probably due also to defence mechanisms occurring during pathological insults such as caries (Vasiliadis et al. 1983; Schüpbach et al. 1992; Nanci, 2007). In particular, DMPI has been shown to have similar functions as dentin sialophosphoprotein and to be detected in primary dentin with a marked decrease in tertiary dentin (Moses et al. 2006).

DMPI has been shown in vitro to facilitate initiation of mineral nucleation at specific sites during bone and dentin formation and to prevent spontaneous calcium phosphate precipitation in areas in which mineralization is not desirable, thus regulating the temporal and spatial aspects of mineral initiation (Narayanan et al. 2001; He et al. 2005). The importance of DMPI in mineralization processes has been well demonstrated by DMPI gene knock-out experiments. Indeed, Ye and coworkers (2004) provided in vivo evidence supporting a role for DMPI in late stages of dentinogenesis, since DMPI is continuously expressed in the dentin of mice after birth. In addition, DMPI null mice exhibited profound defects in the mineralization of dentin, showing the following features: hypomineralization, reduction of dentin thickness, disorganized peritubular areas, enlarged pulp chambers, defects of maturation of predentin to dentin. More recently, Lu and co-workers (2007) clearly demonstrated in transgenic mice that DMPI plays a major role in establishing the proper architecture and organization of the dentin tubular system, either as a consequence of its effects on mineralization or through a direct effect on tubule formation. Indeed, they provided evidence that striking differences were observed in the appearance of dentin tubules from DMP1-null mice compared to controls, with the tubules being disorganized and less branched. These data were in agreement with the ones reported by Butler and co-workers (2002) and Lu and co-workers (2007), which showed by colloidal gold immunostaining that DMPI is predominantly localized in the walls of the rodent dentin tubules.

We have also found some DMPl in the predentin/dentin transition areas (Figure $1 \mathrm{D}$ ) of the examined human teeth, mainly near the mineralization front. These findings are in agreement with the proposal that DMPI has a role in the maturation of predentin matrix as confirmed by the presence of an expanded predentin layer in DMP1-null mice (Ye et al. 2004). Interestingly, the group of Butler and co-workers (Baba et al. 2004) followed changes in distribution of DMPI during the sequential stages of rat molar development. By means of immunohistochemistry and in situ hybridization they found that in 1-day and 1-week old rats, immunoreactions for DMPl were observed in dentinal tubules of mineralized dentin, but not in predentin or odontoblasts. However, after 2 weeks DMPl was observed in dentin tubules, predentin and odontoblasts. At later stages of tooth development, they found many similarities between the distribution of DMPI and that of dentin sialophosphoprotein, both of which are present in dentinal tubules and mature odontoblasts. Our results with fully developed human molars are in agreement with these data. These findings are also partially in agreement with the studies in hypophosphatemic children by Boukpessi and coworkers (2006) and Chaussain-Miller and coworkers (2007). Indeed, they showed positive reaction for $\mathrm{DMPl}$ in odontoblasts and dentin tubules, but only for a short distance within the inner part of the dentin, without reaching the mineralization front.

Limitations of the present study are represented by the poor fixation of the odontoblasts due to the fact that perfusion cannot be performed in human specimens and a limited ultrastructural evaluation, since the examined dentin region was the one within the first 1000-1500 $\mu \mathrm{m}$ from the dental pulp.

In conclusion, within the above mentioned limita- 
tions, the results clearly show for the first time the presence of DMPI in human fully-developed sound molars, showing a strong reaction within the hypermineralized matrix of the peritubular collar delimiting the dentinal tubules, a moderate and diffuse labeling in the intertubular dentin and a weak staining over the proximal predentin region, becoming more intense in distal predentin. The similarity of the present findings with rodent data suggests similarities in dentin formation in the different species. Noteworthy, DMP1 maintains its characteristic localization also in human sound dentin, after complete tooth development. Studies with human teeth comparing the presence and distribution of DMPl in sound dentin (primary and secondary) with that in tertiary dentin, and at early and late stages of the carious process should provide additional insight into its role and potential applications in dentin repair. Furthermore, bone localization of DMP1 and potential signalling role of this molecule will be explored.

\section{Acknowledgements}

The authors wish to thank Mr. Marcello Piccirilli for extensive technical assistance and Mr. Aurelio Valmori for photographical work. We thank Dr. Larry Fisher (National Institute of Dental and Craniofacial Research, Bethesda, MD, USA) for generously donating anti-DMPI (LF122) antibody.

\section{References}

Almushayt A, Narayanan K, Zaki AE, George A. Dentin matrix protein 1 induces cytodifferentiation of dental pulp stem cells into odontoblasts. Gene Ther 2006;13:611-20.

Baba 0, Qin C, Brunna JC, Wygantb JN, McIntyreb BW, Butler WT. Colocalization of dentin matrix protein 1 and dentin sialoprotein at late stages of rat molar development. Matrix Biol 2004; 23:371-79.

Boukpessi T, Septier D, Bagga S, Garabedian M, Goldberg M, ChaussainMiller C. Dentin alteration of deciduous teeth in human hypophosphatemic rickets. Calcif Tissue Int 2006;79:294-300.

Breschi L, Perdigão J, Gobbi P, Mazzotti G, Falconi M, Lopes M. Immunolocalization of collagen type $I$ in etched dentin. J Biomed Mater Res 2003;66A:764-69.

Butler WT, Brunn JC, Qin C, McKee MD. Extracellular matrix proteins and the dynamics of dentin formation. Connect Tissue Res 2002; 43:301-7.

Chaussain-Miller C, Sinding C, Septier D, Wolikow M, Goldberg M, Garabedian M. Dentin structure in familial hypophosphatemic rickets: benefits of vitamin D and phosphate treatment. Oral Dis 2007; 13:482-9.

Feng JQ, Huang $H$, Lu Y, Ye L, Xie Y, Tsutsui TW, et al. The dentin matrix protein 1 (Dmp1) is specifically expressed in mineralized, but not soft, tissues during development. J Dent Res 2003;82:776-80.

Fisher LW, Fedarko NS. Six genes expressed in bones and teeth encode the current members of the SIBLING family of proteins. Connect Tissue Res 2003;44 Suppl 1:33-40.

George A, Sabsay B, Simonian PA, Veis A. Characterization of a novel dentin matrix acidic phosphoprotein. Implications for induction of biomineralization. J Biol Chem 1993;268:12624-30.

Goldberg M, Smith AJ. Cells and extracellular matrices of dentin and pulp: a biological basis for repair and tissue engineering. Crit Rev Oral Biol Med 2004;15:13-27.

Gotliv BA, Robach JS, Veis A. The composition and structure of bovine peritubular dentin: mapping by time of flight secondary ion mass spectroscopy. J Struct Biol 2006;156:320-33.

Jain A, Karadag A, Fohr B, Fisher LW, Fedarko NS. Three SIBLINGs (small integrin-binding ligand, $\mathrm{N}$-linked glycoproteins) enhance factor $\mathrm{H}$ 's cofactor activity enabling MCP-like cellular evasion of complement-mediated attack. J Biol Chem 2002;277:13700-08.

He G, George A. Dentin Matrix Protein 1 immobilized on type i collagen fibrils facilitates apatite deposition in vitro. J Biol Chem 2004;279: 11649-56.

He G, Gajjeraman S, Schultz D, Cookson D, Qin C, Butler WT, et al. Spatially and temporally controlled biomineralization is facilitated by interaction between self-assembled dentin matrix protein 1 and calcium phosphate nuclei in solution. Biochemistry 2005;44:16140-48.

Kulkarni GV, Chen B, Malone JP, Narayanan AS, George A. Promotion of selective cell attachment by the RGD sequence in dentine matrix protein 1. Arch Oral Biol 2000;45:475-84.

Lu Y, Ling Y, Yu S, Zhang S, Xie Y, McKee MD, et al. Rescue of odontogenesis in Dmpl-deficient mice by targeted re-expression of DMP1 reveals roles for $\mathrm{DMPl}$ in early odontogenesis and dentin apposition in vivo. Dev Biol 2007;303:191-201.

Massa LF, Ramachandran A, George A, Arana-Chavez VE. Developmental appearance of dentin matrix protein 1 during the early dentinogenesis in rat molars as identified by high-resolution immunocytochemistry. Histochem Cell Biol 2005;124:197-205.

Moses KD, Butler WT, Qin C. Immunohistochemical study of small integrin-binding ligand, $\mathrm{N}$-linked glycoproteins in reactionary dentin of rat molars at different ages. Eur J Oral Sci 2006;114:216-22.

Nanci A. In: Ten Cate's oral histology: development, structure and function. 7th edn. St Louis, Missouri: Mosby, 2007, pp. 191-238.

Narayanan K, Srinivas R, Ramachandran A, Hao J, Quinn B, George A. Differentiation of embryonic mesenchymal cells to odontoblast-like cells by overexpression of dentin matrix protein 1. Proc Natl Acad Sci USA 2001;98:4516-21.

Narayanan K, Gajjeraman S, Ramachandran A, He G, Park KW, Hao J, et al. Dual Functional Roles of Dentin Matrix Protein 1. J Biol Chem 2003;278:17500-8.

Narayanan K, Gajjeraman S, Ramachandran A, Hao J, George A. Dentin Matrix Protein 1 regulates dentin sialophosphoprotein gene transcription during early odontoblast differentiation. J Biol Chem 2006; 281:19064-71.

Smith $A J$, Lesot $H$. Induction and regulation of crown dentinogenesis: embryonic events as a template for dental tissue repair? Crit Rev Oral Biol Med 2001;12:425-37.

Suppa P, Ruggeri A Jr, Tay FR, Prati C, Biasotto M, Falconi M, et al. Reduced antigenicity of type I collagen and proteoglycans in sclerotic dentin. J Dent Res 2006;85:133-7.

Schüpbach P, Lutz F, Guggenheim B. Human root caries: histopathology of arrested lesions. Caries Res 1992;26:153-64.

Toyosawa S, Kanatani N, Shintani S, Lobata M, Yuki M, Kishino M, et al. Expression of dentin matrix protein 1 (DMP1) during fracture healing. Bone 2004;35:553-61.

Qin C, D'Souza R, Feng JQ. Dentin matrix protein 1 (dmpl):new and important roles for biomineralization and phosphate homeostasis. J Dent Res 2007;86:1134-41.

Vasiliadis L, Darling AI, Levers BG. The histology of sclerotic human root dentine. Arch Oral Biol 1983;28:693-700.

Weiner S, Veis A, Beniash E, Arad T, Dillon JW, Sabsay B, et al. Peritubular dentin formation: crystal organization and the macromolecular constituents in human teeth. J Struct Biol 1999;126:27-41.

Ye L, Macdougall M, Zhang S, Xie Y, Zhang J, Li Z, et al. Deletion of Dentin Matrix Protein-1 leads to a partial failure of maturation of predentin into dentin, hypomineralization, and expanded cavities of pulp and root canal during postnatal tooth development. J Biol Chem 2004;279:19141-8. 